\title{
Learning to be in control involves response-specific mechanisms
}

\author{
Marit F. L. Ruitenberg ${ }^{1} \cdot$ S. Braem ${ }^{1,2} \cdot$ H. Du Cheyne ${ }^{1} \cdot$ W. Notebaert $^{1}$ \\ Published online: 9 May 2019 \\ (C) The Psychonomic Society, Inc. 2019
}

\begin{abstract}
Conflict adaptation refers to our ability to modulate our attention in line with changing situational demands, so we can engage in goal-directed behavior. While there is ample evidence demonstrating that such adaptation in conflict tasks can be captured using different response modalities, it remains unknown whether these effects rely on domain-general mechanisms applied to different response modalities, or are the result of more inherently response-specific processes. Here, we used an individual-differences approach to evaluate whether conflict adaptation in two highly similar tasks using different response modalities are related. Specifically, participants performed two versions of a Stroop task, one in which they responded via key presses and one in which they responded via mouse movements. In both tasks, we manipulated the item-specific proportion of (in)congruent trials ( $80 \%$ vs. $20 \%$ congruent). This allowed us to evaluate the item-specific proportion congruency (ISPC) effect, a hallmark indicator of conflict adaptation. ISPC effects were observed in both response modalities. However, we found no indications that individual differences in the ISPC effects of the two response modalities were related. This raises the question whether findings from studies on conflict adaptation measured by different modalities can reliably be compared. Furthermore, these results suggest that response modality plays a more integrative role in these adaptive processes, rather than being the mere output of a domaingeneral control mechanism. This is consistent with contingency learning accounts of the ISPC effect and associative learning models of cognitive control.
\end{abstract}

Keywords Cognitive control $\cdot$ Stroop task $\cdot$ Item-specific proportion congruency $\cdot$ Response modalities $\cdot$ Mouse tracking

\section{Introduction}

Imagine you are carrying a birthday cake, when suddenly it slips out of your hand - most likely you will try to catch and save the cake. However, would you have been carrying a tray with cups of hot tea and coffee instead, you probably would not attempt to catch these as you could hurt yourself. This example illustrates people's ability to suppress a relatively automatic action in favor of a more appropriate one, in order to engage in appropriate, goal-direct behavior (Botvinick,

Marit F. L. Ruitenberg

marit.ruitenberg@ugent.be

1 Department of Experimental Psychology, Faculty of Psychology and Educational Sciences, Ghent University, Henri Dunantlaan 2, 9000 Ghent, Belgium

2 Department of Experimental and Applied Psychology, Vrije Universiteit Brussel, Brussels, Belgium
Cohen, \& Carter, 2004; Verguts \& Notebaert, 2008). In addition, we are able to flexibly update our cognitive control settings, allowing us to adapt ongoing actions to changing situational demands. These processes are often referred to as conflict adaptation, which is considered a core aspect of human cognitive control (Botvinick, Braver, Barch, Carter, \& Cohen, 2001; Notebaert \& Verguts, 2008; Verguts \& Notebaert, 2009). To investigate conflict adaptation in the lab, researchers often use conflict paradigms such as the Stroop task, Simon task, and Eriksen flanker task, where relevant and irrelevant stimulus features (e.g., ink color and word meaning in the Stroop task) trigger responses that are either the same without causing conflict (i.e., congruent trials) or in competition with each other yielding conflict (i.e., incongruent trials). People typically perform worse on incongruent than congruent trials.

Crucially, this so-called congruency effect can be modulated by contextual factors. For example, the congruency effect is typically reduced directly after incongruent as compared to congruent trials (i.e., congruency sequence effect; for a review, see Duthoo, Abrahamse, Braem, Boehler, \& 
Notebaert, 2014), or in contexts where there are more incongruent trials than congruent trials relative to a context with more congruent than incongruent trials (i.e., proportion congruency effect; for a review, see Bugg \& Crump, 2012). These effects are often explained by the conflict-monitoring hypothesis (Botvinick et al., 2001), which proposes that participants monitor conflict and modulate attention to maintain appropriate performance. More specifically, according to this hypothesis the anterior cingulate cortex monitors the amount of conflict in the environment, and signals regions like the dorsolateral prefrontal cortex to increase its top-down influence on performance when high levels of conflict are detected (e.g., in a mostly incongruent context). As a result, goaldirected behavior is preserved in situations where multiple incongruent response tendencies emerge.

The observation that people adapt to conflict frequency has also been shown at the level of individual items. For example, the congruency effect is typically reduced for items that are mostly presented in an incongruent manner as compared to items that are mostly presented in a congruent manner (i.e., item-specific proportion congruency [ISPC] effect; Jacoby, Lindsay, \& Hessels, 2003). The conflict-monitoring hypothesis cannot account for this effect (Bugg \& Crump, 2012), as ISPC designs typically balance the number of congruent and incongruent items across the experiment such that conflict occurs on $50 \%$ of trials overall. The observation of differential congruency effects for different items is therefore difficult to explain by a model that proposes a global level of top-down control across the experiment. This has led others to develop computational models and theories with emphasis on the close integration between (implicit) learning and conflict adaptation (e.g., Abrahamse, Braem, Notebaert, \& Verguts, 2016; Egner, 2014; Verguts \& Notebaert, 2008, 2009). From these frameworks, it follows that cognitive control strategies are contextsensitive, both in relation to their input (i.e., item-sensitivity) and to their output (i.e., response modalities). While the former has been the topic of many studies (for recent reviews, see Braem, Abrahamse, Duthoo, \& Notebaert, 2014; Egner, 2014), the role of response modalities in conflict control strategies has gained little attention thus far.

Congruency effects and conflict adaptation effects have been demonstrated in a variety of response modalities. For example, the Stroop effect has been shown with key presses and verbal responses (for reviews, see Laird et al., 2005; MacLeod, 1991) as well as with mouse movements (e.g., Incera, Markis, \& McLennan, 2013). Similarly, the (itemspecific) proportion congruency effect has been observed both with key presses (e.g., Abrahamse, Duthoo, Notebaert, \& Risko, 2013; Grandjean et al., 2013) and with mouse movements (e.g., Bundt, Ruitenberg, Abrahamse, \& Notebaert, 2018; Ruitenberg, Abrahamse, Santens, \& Notebaert, 2019). However, from these studies it remains unclear whether such control relies on mechanisms that are response-specific, or on more domain-general strategies instead. A few studies did investigate transfer of the congruency sequence effect between response modalities. For example, Braem, Verguts, and Notebaert (2011) showed that the congruency sequence effect could be observed between a vertical and horizontal conflict task only when participants used two highly similar response sets (i.e., both manual), and not when they used two distinct response sets (i.e., hand vs. foot responses; see also, Janczyk \& Leuthold, 2018). While these studies already suggest that cognitive control may at least to some extent be response-specific (but see Weissman, Colter, Drake, \& Morgan, 2015), the response-specificity could also have been elicited by the specific task demands of these studies (i.e., foot and hand response trials were randomly intermixed and thus in constant competition), or could be specific to these more transient conflict adaptation effects (i.e., congruency sequence effect vs. proportion congruency effects). Therefore, this present study took another approach, and evaluated whether individual differences in the ISPC effect are related across different response modalities. Specifically, the present study used the Stroop task to examine whether across participants the magnitude of the ISPC effect as reflected in key-press responses was correlated with the magnitude of the ISPC effect as reflected in the various mouse movement parameters. In the key-press version of the Stroop task we measured participants' reaction time (RT) on each trial. In the mouse-tracking version, we measured their initiation time (IT), movement time (MT), and the area under the curve (AUC) of their empirical trajectory relative to the ideal trajectory.

In line with previous research, we expect to observe a reliable and stable ISPC effect in both key presses (e.g., Grandjean et al., 2013) and mouse movements (e.g., Bundt et al., 2018). In a similar vein, recent studies reported positive correlations between cognitive control indices measured at two separate test sessions over a 2-month interval, demonstrating the reliability of control strategies within tasks across time (Clayson \& Larson, 2013; Feldman \& Freitas, 2016). On the other hand, other studies found that individual differences in the size of the congruency sequence effect were uncorrelated across Simon, flanker, and/or Stroop tasks (Keye, Wilhelm, Oberauer, \& van Ravenzwaaij, 2009; Keye, Wilhelm, Oberauer, \& Stürmer, 2013; Whitehead, Brewer, \& Blais, 2019), suggesting that control strategies differ between tasks and may be input-specific (item-sensitivity). However, as the conflict tasks in Keye et al.'s and Whitehead et al.'s studies all required manual key press responses, the role of response modality (i.e., output) remains unknown. While there are indications that the relative size of cognitive control indices may be sensitive to the response modality that is used (Donohue, Liotti, Perez, \& Woldorff, 2012; Weekes \& Zaidel, 1996), these studies compared control at the group level and did not examine individual differences. As such, they do not allow for making inferences on the potential response-specificity of cognitive control strategies. 
Importantly, our individual-differences approach would allow us to examine conflict adaptation across response modalities. If a domain-general mechanism exists that is relatively independent of response type (cf. conflict-monitoring hypothesis), the ISPC effect is expected to be stable across response modalities and we would observe a significant correlation between the ISPC effects in the key-press and mousetracking tasks. In contrast, if different control mechanisms drive performance depending on the response modality (cf. associative learning models), we should observe that the magnitude of the ISPC effects in both tasks are uncorrelated; this would argue in favor of response-specific control mechanisms. While we will also calculate the correlation between both Stroop effects, we focus on the ISPC effect as this is regarded a purer measure of cognitive control than the main congruency effect, which reflects selective attention abilities rather than adaptive adjustments in control (Verguts \& Notebaert, 2008).

\section{Method}

\section{Participants}

A total of 63 psychology students (seven males; mean age $18.44 \pm 1.30$ years) of Ghent University participated in the current study. Prior work showed strong correlations for conflict adaptation measures across time (mean $r=.54$, Clayson \& Larson, 2013; Feldman \& Freitas, 2016). However, we aimed to include a minimum of 60 participants to be able to detect a correlation of medium to strong effect size. For example, sample size calculation using $\mathrm{G}^{*}$ Power v3.1.9.2 software for a correlation of 0.35 (Cohen, 1988) with a one-sided alpha level of 0.05 and statistical power of 0.80 suggested we required 49 participants. All participants were native Dutch speakers, were right-handed according to Annett's Handedness Inventory (Annett, 1970), and had normal or corrected-to-normal vision. In addition, none reported to be dyslexic or color-blind. All participants provided written informed consent before the start of the experiment and received partial course credit for their participation. The experiment adhered to the general ethical protocol of the ethics committee of the Faculty of Psychology and Educational Sciences of Ghent University.

\section{Apparatus}

Stimulus presentation and data registration were controlled by MouseTracker (Freeman \& Ambady, 2010) and E-prime (C) 2.0 software for the mouse-tracking and key-press versions of the task, respectively. Participants performed the mousetracking version of the task with a standard Dell computer mouse with the cursor speed set at the 6/11 default mode in Windows 7. Key-press responses were given on a standard qwerty computer keyboard. The experiments ran on a Dell Optiplex 3020 mini-tower connected to a Benq XL2411Z LED monitor $(1,920 \times 1,080)$.

\section{Experimental tasks and procedure}

Upon entering the lab, participants were informed about the procedure of the study and told that they would perform two tasks during the experimental session. Before starting these, they first completed a short questionnaire regarding their demographic details and filled out the Handedness Inventory to check for right-handedness. After completing the questionnaires and providing written informed consent, participants sat in front of a computer screen on which the instructions of the first task were presented.

The Stroop stimuli in the present study consisted of eight color words (red, yellow, green, blue, violet, orange, pink, brown) that were used as words as well as ink colors (the Dutch words were "rood," "geel," "groen," "blauw," "paars," "oranje," "roze," "bruin"). Two different subsets of four distinguishable colors each were created to avoid overlap in colors between the two versions of the Stroop task (set 1: red-yellow-violet-brown, set 2: green-blue-pink-orange; cf. Geukes, Gaskell, \& Zwitserlood, 2015). The two subsets were identical for all participants, but the assignment of the subsets to the two versions of the Stroop task was counterbalanced across participants. Within each color set, colors were paired (red and yellow, violet and brown, green and orange, blue and pink), so that on incongruent trials the response boxes relating to the task-relevant and task-irrelevant stimulus features were on opposite sides of the start location; far-left was always linked to far-right, and center-left was always linked to center-right. This pairing prompted participants to commit to moving the mouse cursor to either the left or right side of the screen, which optimized the tracking of conflict processing as reflected in mouse trajectories (cf. Bundt et al., 2018; Ruitenberg et al., 2019). For one of the pairs of a color set, $80 \%$ of the trials were congruent, while for the other pair of that color set only $20 \%$ of the trials were congruent. For example, for color set 1 the words red and yellow were shown in their congruent ink color in $80 \%$ of the trials, while the words violet and brown were only shown in their congruent ink color in $20 \%$ of the trials. This proportion was counterbalanced across participants and allowed us to examine the ISPC effect. An index for this effect can be obtained by subtracting the difference between incongruent trials and congruent trials in the $20 \%$ congruent condition from the difference between incongruent and congruent trials in the $80 \%$ congruent condition.

As aforementioned, participants completed two versions of the Stroop task: one in which they responded via mouse movements, and one in which they responded via key presses. In the mouse-tracking version of the task, each trial started 
with the presentation of a start button $(2 \times 5.5 \mathrm{~cm})$ in the central bottom of the screen and four response boxes $(5 \times 5$ $\mathrm{cm}$ ) horizontally aligned at the top of the screen (Bundt et al., 2018; Ruitenberg et al., 2019). As illustrated in Fig. 1 (left side), each response box was a black square outlined in white, in which a white number was presented (from left to right " 1 ," " 2 ," " 3 ," and " 4 "). Each of these numbers corresponded with a color. When participants clicked on the start button, this button disappeared and a Stroop stimulus was presented in the center of the display (Arial font, height $1.5 \mathrm{~cm}$ ). Upon presentation of the stimulus, the mouse cursor was automatically relocated to the center of the start button so that all mouse movements started from exactly the same location. The distance between the start location of the mouse cursor and each response box was $28 \mathrm{~cm}$. A trial ended when the participant clicked a response box. If the participant clicked the wrong response box, a red " $\mathrm{X}$ " appeared in the middle of the display for 2,000 ms. After each trial, a black screen was presented for $500 \mathrm{~ms}$ before the next trial could be started. If participants had not yet started a mouse movement within 2,000 ms after stimulus presentation, a message was presented telling the participant to respond more rapidly next time (cf. Faulkenberry, Cruise, Lavro, \& Shaki, 2016; Freeman \& Ambady, 2009; Yamamoto, Incera, \& McLennan, 2016).

The key-press version of the Stroop-task was almost identical to the mouse-tracking version, except that participants were instructed to respond to the ink color of the stimulus by pressing one of four keys on the keyboard (c, v, b, or n) with the fingers of their right hand. Each key corresponded to a particular color. Each trial started with a black screen on which a white fixation cross was centrally presented (Fig. 1, right side). This fixation cross was a sign to the participant to press the space bar of the keyboard with the left hand or the thumb of the right hand (since the other fingers of their right hand were placed on the response keys of the keyboard, as instructed). After pressing the space bar, the fixation cross disappeared and a stimulus was shown in the center of the display. A trial ended after the participants pressed one of

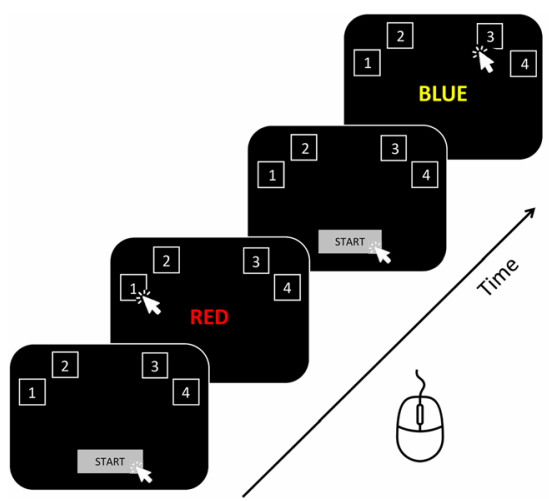

Fig. 1 An overview of the stimulus presentation in the Stroop task with mouse movements (left) and key presses (right). Note that all words were actually displayed in Dutch and that there was an inter-trial interval of 500 the four keys. When they pressed the wrong key, a red " $\mathrm{X}$ " was shown for $2,000 \mathrm{~ms}$ in the middle of the screen. The intertrial interval was $500 \mathrm{~ms}$.

Each version of the task started with a practice block of 16 randomly ordered congruent and incongruent trials (50\% congruent). During this practice block, participants were presented a sheet of paper showing the four response options and their corresponding colors. Hence, participants could check this paper to find out which response box or key corresponded with which color. When the practice block was finished the researcher removed the color paper. Participants did not experience difficulties remembering the correct combinations. After the practice block, the participants performed four experimental blocks. Each block consisted of 160 randomly ordered trials, with the restriction that a trial could not require the same response (and thus have the same ink color) as the previous one. Participants could take a self-paced break between the blocks. The order of the key-press and mouse-tracking versions of the tasks was counterbalanced across participants and the total duration of the experiment was approximately $1 \mathrm{~h}$.

\section{Data processing}

The MouseTracker software recorded the real-time $\mathrm{x}$, y coordinates of the computer mouse every 13-16 ms $(\sim 70 \mathrm{~Hz})$. Using MouseTracker Analyzer, the raw trajectory data of the mouse movements were rescaled to a standard coordinate space ( $\mathrm{x}$-axis range -1 to $1 ; \mathrm{y}$-axis range 0 to 1.5 ) as well as remapped to the right. The data were then time-normalized by the software into 101 time-steps, with each of these time-steps having a normalized $\mathrm{x}$ and $\mathrm{y}$ coordinate. This makes it possible to compare responses that vary in duration (and thus have a different amount of sampled coordinates) between conditions (see Freeman \& Ambady, 2010). Subsequently, we determined the IT, MT, and AUC to evaluate participants' performance. The IT is defined as the time from stimulus presentation until movement onset. MT refers to the total time from the mouse-movement onset to the click on the response box.

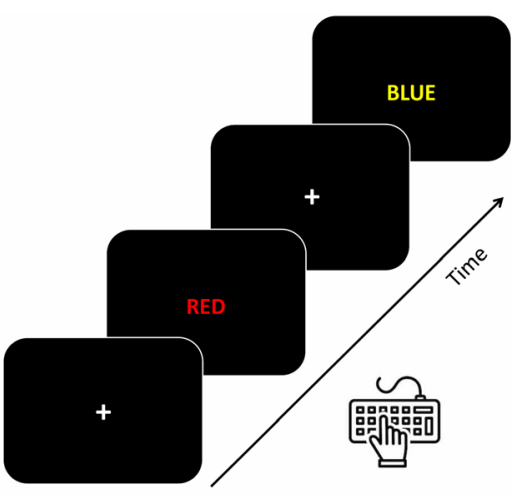

ms. The figure shows examples of both a congruent ("RED" in red ink) and an incongruent trial ("BLUE" in yellow ink; color figure online) 
The AUC is the geometric area between the participants' empirical response trajectory and the ideal trajectory (i.e., a straight line from the start location of the mouse cursor to the correct response box). It reflects the overall level of processing difficulty, with a greater area indicating more difficult processing. For the key-press data, we measured the RT, defined as the time between stimulus presentation and pressing a key. For both versions of the task, we also determined the proportion of incorrect responses. Each dependent variable was calculated as a function of proportion ( $80 \%$ vs. $20 \%)$ and congruency (congruent vs. incongruent) condition.

Further data-processing was performed using SPSS software (version 23.0; IBM Corp, 2015). For both the mousetracking data and the key-press data the first trial of each block and error and post-error trials were excluded. We also excluded trials when the MT of mouse tracking or the RT of key presses exceeded the mean MT or RT by more than 3 standard deviations. This was done per participant and separately for each proportion and congruency condition, and resulted in a removal of $1.96 \%$ of the trials in the mouse-tracking version and $2.16 \%$ of the trials in the key-press version of the task. We also used SPSS to perform all statistical analyses, except for additional Bayesian analyses, which were performed using JASP software (version 0.8.1; JASP Team, 2017).

\section{Results}

In the results section, we first evaluate whether the ISPC effect was reflected in both the key-press (RT) and mouse-tracking (IT/MT/AUC) versions of our Stroop task. We then test the consistency of this effect within each version of the task. Most importantly, we test whether individual differences in magnitude of the ISPC effect were associated between both tasks. To this end, we ran correlation analyses using both the more traditional null-hypothesis significance testing (NHST) approach and a Bayesian approach. As associative learning models predict the absence of a significant correlation, we used this latter approach to be able to determine whether our data provided evidence in favor of the null or alternative hypothesis in case NHST results showed a non-significant correlation. We also ran each of these analyses on the error rates in each version of the task. Finally, to address all control phenomena in the current Stroop task, we evaluate whether simple congruency (i.e., Stroop) effects could be observed and whether these were correlated within and across tasks.

\section{Conflict adaptation}

We ran separate repeated-measures ANOVAs on RT for the key-press data and on IT, MT, and AUC for the mouse-tracking data with Proportion (2;80\% vs. 20\%) and Congruency (2; congruent vs. incongruent) as within-subject variables. For the key-press version of the Stroop task, results of the ANOVA on RT showed a main effect of Congruency, $F(1,62)=166.22, p<.001, \eta_{p}{ }^{2}=.73$. Participants responded slower in the incongruent than the congruent condition ( $885 \mathrm{vs} .788 \mathrm{~ms}$ ), which is in line with the typical Stroop effect. Moreover, results showed a significant Proportion $\times$ Congruency interaction, $F(1,62)=116.06, p<.001, \eta_{p}{ }^{2}=.65$. Individual post hoc ANOVAs for each Proportion condition showed that the RT difference between congruent and incongruent trials was significant both for $80 \%$ congruent items (757 vs. $916 \mathrm{~ms}), F(1,62)=234.83, p<.001, \eta_{p}{ }^{2}=.79$, and for $20 \%$ congruent items ( 819 vs. $855 \mathrm{~ms}), F(1,62)=17.91, p<.001$, $\eta_{p}{ }^{2}=.22$. As Fig. 2 (panel A) shows, the congruency effect was larger for the $80 \%$ compared to the $20 \%$ congruent condition, thus demonstrating the ISPC effect for the keypress version of the Stroop task.

Figure 3 shows the mouse movement trajectories of congruent and incongruent trials for the $80 \%$ congruent and $20 \%$ congruent items. Results of the ANOVA on IT showed neither significant main effects of Congruency, $F(1,62)<1, p=.384, \eta_{p}{ }^{2}=.01$, and Proportion, $F(1,62)<1$, $p=.774, \eta_{p}{ }^{2}<.01$, nor a significant interaction effect (Fig. 2 , panel $\left.\mathrm{B} ; F(1,62)=2.33, p=.132, \eta_{p}{ }^{2}=.04\right)$. For MT, results showed a main effect of Congruency, $F(1,62)=158.03, p<.001, \eta_{p}{ }^{2}=.72$. Participants were slower at performing mouse movements in the incongruent condition than in the congruent condition (1,240 vs. $1,133 \mathrm{~ms}$ ), thus illustrating the Stroop effect. Crucially, results also showed a significant Proportion $x$ Congruency interaction, $F(1,62)=66.68, p<.001, \eta_{p}{ }^{2}=.52$. Individual post hoc ANOVAs showed that the difference in MT between congruent and incongruent trials was significant both for $80 \%$ congruent items (1,116 vs. 1,281 $\mathrm{ms}), F(1,62)=157.46, p<.001, \eta_{p}{ }^{2}=.72$, and for $20 \%$ congruent items ( 1,151 vs. $1,198 \mathrm{~ms}), F(1,62)=29.94, p<.001$, $\eta_{p}{ }^{2}=.32$. As Fig. 2 (panel C) shows, the difference in performance between congruent and incongruent trials was larger for the $80 \%$ than for the $20 \%$ congruent condition, indicative of the ISPC effect.

For AUC, we also observed a main effect of Congruency, $F(1,62)=122.43, p<.001, \eta_{p}{ }^{2}=.66$. Specifically, the AUC was larger for incongruent than for congruent trials $(0.71$ vs. 0.49$)$, again illustrating the Stroop effect. Results additionally showed that movements trajectories deviated more from the optimal trajectory for $80 \%$ than $20 \%$ congruent trials (.63 vs. .56), $F(1,62)=10.23, p=.002, \eta_{p}{ }^{2}=.14$. Importantly, we again observed a significant Proportion $\times$ Congruency interaction for AUC, $F(1,62)=28.25, p<.001, \eta_{p}{ }^{2}=.31$ (Fig. 2, panel D). Individual post hoc ANOVAs showed that the difference between congruent and incongruent trials was significant both for $80 \%$ congruent items ( 0.48 vs. 0.79 ), 


\section{a Key presses RT}

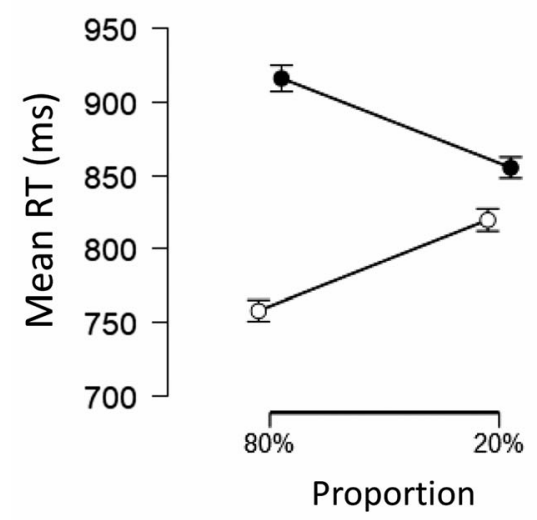

c Mouse movements MT

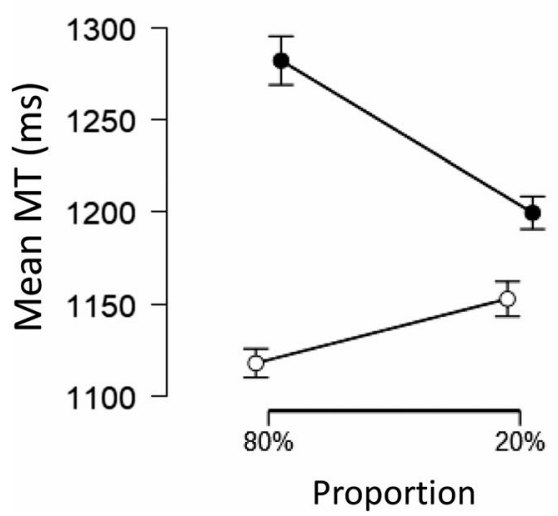

Fig. 2 Mean reaction time (RT) in the key-press task (panel A), and mean initiation time (IT), movement time (MT), and the area under the curve (AUC) in the mouse-tracking task (panels B-D) for congruent and

$F(1,62)=91.36, p<.001, \eta_{p}{ }^{2}=.59$, and for $20 \%$ congruent items $(0.50$ vs. 0.63$), F(1,62)=60.37, p<.001, \eta_{p}{ }^{2}=.49$. Here, too, the data were in line with the ISPC effect: the congruency effect was larger for the $80 \%$ congruent condition compared to the $20 \%$ congruent condition. Overall, these results demonstrate the ISPC effect for the execution of responses in the mouse-tracking version of the task.

\section{Internal consistency of the ISPC effect}

To evaluate whether individual differences in the observed ISPC effects were consistent within each response modality, we determined the correlation between the size of the ISPC effect in odd and even blocks (B1+3 vs. B2+4) for each version of our Stroop task. The ISPC effect was calculated by subtracting the difference in performance between incongruent and congruent trials on $20 \%$ congruent items from the difference on $80 \%$ congruent items. ${ }^{1}$ Besides the more

\footnotetext{
${ }^{1}$ We also repeated our analyses on standardized ISPC scores controlling for overall differences in mean performance. Results yielded the same pattern of results as reported for the unstandardized data.
}

\section{b Mouse movements IT}

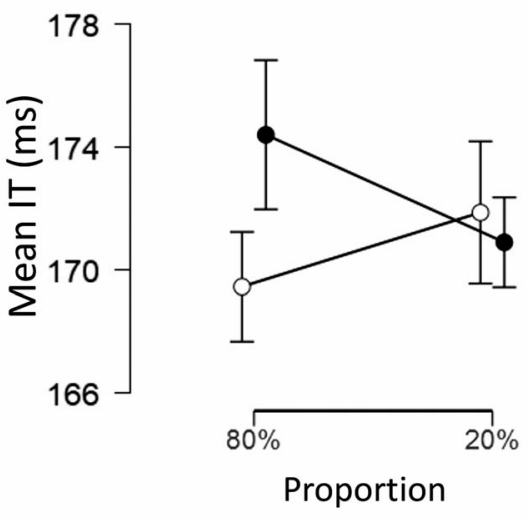

\section{d Mouse movements AUC}

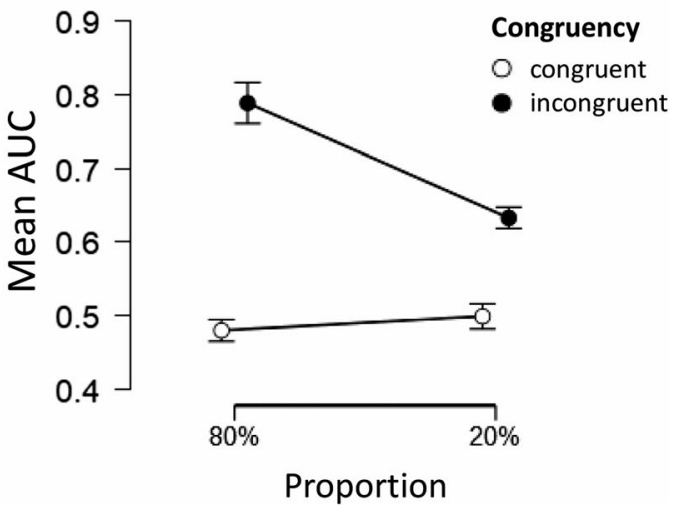

incongruent trials as a function of proportion congruency. Except for IT, all panels show a significant item-specific proportion congruency (ISPC) effect. Error bars represent standard errors

traditional NHST approach, we also determined the Bayes factor $\left(\mathrm{BF}_{10}\right)$ using a Bayesian correlation analysis with default prior settings. $\mathrm{A} \mathrm{BF}_{10}$ larger than 3 is regarded as moderate evidence for the alternative hypothesis (with $\mathrm{BF}_{10}>10$ being strong evidence), and a $\mathrm{BF}_{10}$ between 1 and 3 as merely anecdotal evidence. In contrast, a $\mathrm{BF}_{10}$ smaller than 0.33 reflects moderate evidence for the null hypothesis (with $\mathrm{BF}_{10}<0.1$ being strong evidence), and a $\mathrm{BF}_{10}$ between 1 and 0.33 reflects anecdotal evidence for the null hypothesis (Dienes, 2011; Nuzzo, 2017).

Figure 4 shows the relationship between the ISPC effects in the odd and even blocks of each task as well as the results of the traditional and Bayesian correlation analyses for RT, MT, and AUC. For the key-press version of the task, results of the NHST approach showed a significant, positive correlation for the ISPC effect as reflected in RT between odd and even blocks (Fig. 4, panel A). In addition, the Bayesian approach showed strong evidence in favor of the alternative hypothesis that the individual differences were correlated. For the mousetracking version, results revealed similar patterns for the ISPC effect as reflected in MT and AUC (Fig. 4, panels B and C). Using the NHST approach, results showed a significant, 

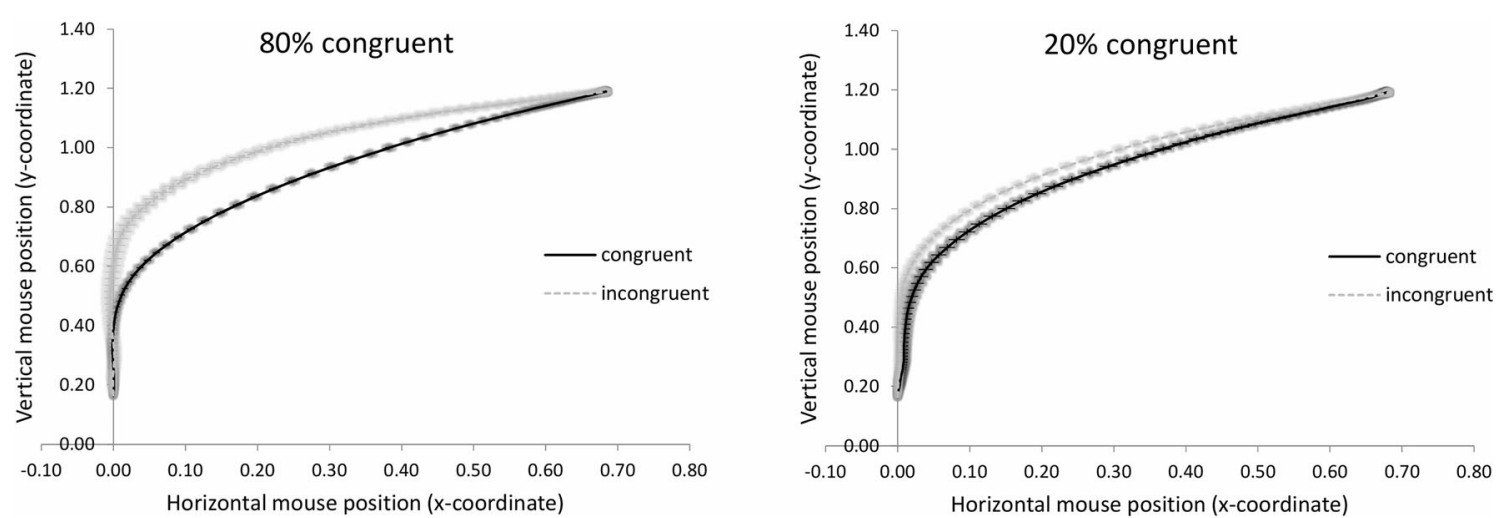

Fig. 3 Mean mouse trajectories for the congruent and incongruent trials as a function of proportion congruency. Error bars represent $95 \%$ confidence intervals

positive correlation for both MT and AUC. Results of the Bayesian approach indicated that the current data provide strong to extreme evidence for consistent ISPC effects reflected in MT and AUC. We did not find evidence for an association in IT, which is unsurprising given the absence of an ISPC effect in this parameter in the first place $(r=-.015$, $p=.907 ; \mathrm{BF}_{10}=0.15$ ). These results suggest that individual differences in the ISPC effect are consistent within each response modality.

\section{Correlations between response modalities}

To evaluate whether individual differences in magnitude of the observed ISPC effect were associated between both tasks, we again performed correlation analyses. For each combination of the ISPC as reflected in RT and our mouse-movement parameters, we determined both the Pearson correlation and the Bayes factor $\left(\mathrm{BF}_{10}\right)$ using a Bayesian correlation analysis with default prior settings. Note that these analyses included double the amount of observations compared to the above-mentioned internal consistency measurements, resulting in better point estimates of our ISPC effect. Figure 5 illustrates the relationships between the ISPC effects as reflected in our parameters and shows the results of the traditional and Bayesian correlation analyses. Using the NHST approach, we observed no significant correlations between magnitude of the ISPC effects in both versions of the Stroop task ( $p s>$.141). Results of the Bayesian approach indicated that the $\mathrm{BF}_{10} \mathrm{~s}$ for the correlations ranged between 0.16 and 0.45 . This indicates that the current data provide anecdotal to moderate evidence in favor of the null hypothesis that there is no correlation between individual differences in the size of the ISPC effect in key-press (RT) and mouse-movement (IT/MT/AUC) parameters. These results suggest that individual differences in the ISPC effect are relatively independent across response modalities.

\section{Error rates}

We also evaluated performance in terms of the error rates by performing separate ANOVAs on the proportion of errors in each task with Proportion (2) and Congruency (2) as withinsubject variables. For the key-press version of the task, results showed that participants made more errors on incongruent than congruent trials ( 0.07 vs. 0.06$), F(1,62)=12.30, p=.001$, $\eta_{p}{ }^{2}=.17$. Results further showed a significant Proportion $\times$ Congruency interaction, $F(1,62)=23.47, p<.001, \eta_{p}^{2}=.27$. As illustrated in Fig. 6 (panel A), participants made more errors on incongruent than congruent trials in the $80 \%$ congruent condition, $F(1,62)=26.69, p<0.001, \eta_{p}{ }^{2}=.30$. The difference in the $20 \%$ congruent condition was not significant, $F(1,62)=1.52, p=.222, \eta_{p}{ }^{2}=.02$. Results of the ANOVA on error rates in the mouse-tracking version of the task also showed that participants made more errors in the incongruent condition than in the congruent condition ( 0.025 vs. 0.019$)$, $F(1,62)=9.48, p=.003, \eta_{p}{ }^{2}=.13$. There was also a significant Proportion $\times$ Congruency interaction, $F(1,62)=5.81, p=.019$, $\eta_{p}{ }^{2}=.09$. Post hoc ANOVAs showed that participants made more errors on incongruent trails compared to congruent trials in the $80 \%$ congruent condition, $F(1,62)=13.27, p=.001$, $\eta_{p}{ }^{2}=.17$, but that the difference in the $20 \%$ congruent condition was not significant $\left(F<1, p=.704, \eta_{p}^{2}<.01\right.$; see Fig. 6 , panel B).

When evaluating the internal consistency of the ISPC effect as reflected in errors, results of the NHST approach showed that the within-task correlation (odd vs. even blocks) was not significantly correlated in either version of the task, $|r s(63)|<.21, p s>.097$, suggesting an already poor internal consistency. The Bayesian approach showed moderate to anecdotal evidence in favor of the null hypothesis for the key-press $\left(\mathrm{BF}_{10}=0.29\right)$ and mousetracking versions $\left(\mathrm{BF}_{10}=0.60\right)$, respectively. Still, for the sake of completeness, we also ran correlation analyses on the magnitude of the ISPC effects across both versions of the task to evaluate whether there was an association 
a Key presses RT

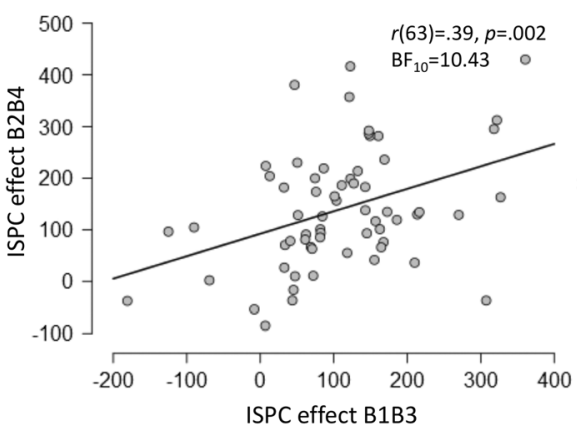

b Mouse movements MT

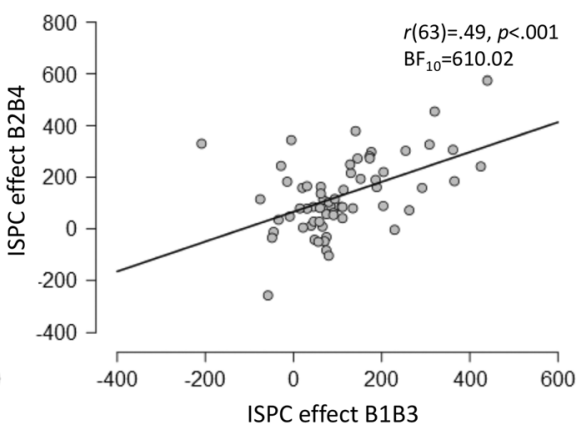

C Mouse movements AUC

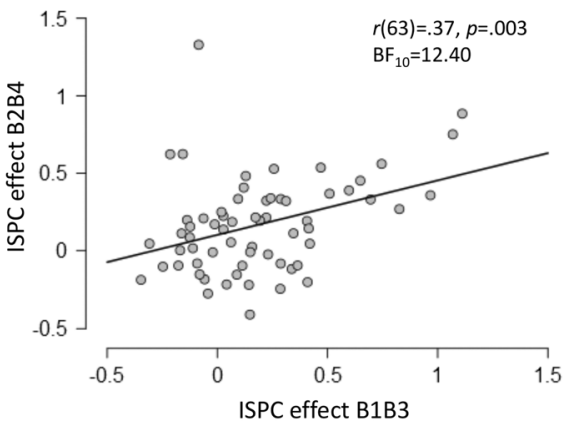

Fig. 4 Scatterplots illustrating the relationship between the item-specific proportion congruency (ISPC) effects as reflected in the odd and even blocks of the key-press version of the task (reaction times (RTs); panel A) and the mouse-tracking task version of the task (movement time (MT) and the area under the curve (AUC); panels B and C, respectively)

differences in the observed effects were consistent within each response modality. Results showed significant correlations for each of our four dependent variables, $r s(63)>.33, p s<.008$, $\mathrm{BF}_{10} \mathrm{~S}>4.92$ (with $p s<.001$ and $\mathrm{BF}_{10} \mathrm{~s}>100$ for RT, MT, and AUC), suggesting that individual differences in the congruency effect are consistent within each response modality. However, we found no evidence that these individual differences were correlated across both versions of the task $(r s(63)$ between - .20 and $.26, p s>.052 ; \mathrm{BF}_{10}$ s between 0.52 and 0.99 ). Taken together, similar to our aforementioned ISPC findings, these results suggest that individual differences in the congruency effect are consistent within each response modality but relatively independent across modalities.

Finally, we tested whether the congruency effect could also be observed in our data and if individual differences in this measure were correlated across the two Stroop tasks. Consistent with the congruency effect, performance was significantly worse on incongruent compared to congruent trials for RT $(\Delta=97 \mathrm{~ms}), \operatorname{MT}(\Delta=91 \mathrm{~ms})$, and $\operatorname{AUC}(\Delta=.18)$, $F s(1,62)>103.81, p s<.001, \eta_{p}{ }^{2} s>.62$. There was no significant difference in IT $(\Delta=2 \mathrm{~ms}), F(1,62)<1, p=.390$. Like we did for the ISPC effect, we determined the correlation between the size of the congruency effect in odd and even blocks for each version of our Stroop task to evaluate whether individual

\section{Discussion}

The present study examined whether conflict control processes are the result of a domain-general conflict adaptation process, or are rather specific to the response modalities used to perform the task. Using an individualdifferences approach, we evaluated whether conflict adaptation as reflected in the ISPC effect was correlated across

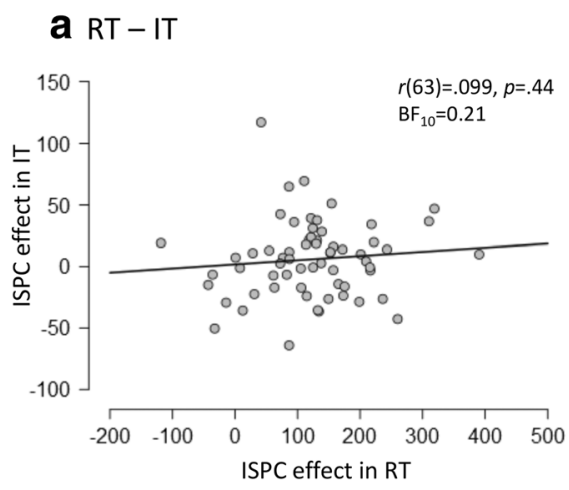

b $\mathrm{RT}-\mathrm{MT}$

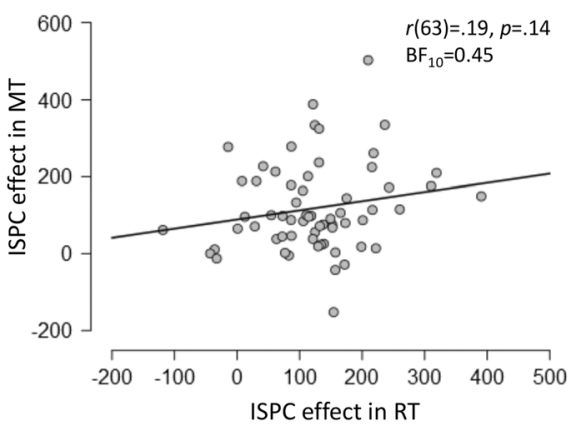

C RT - AUC

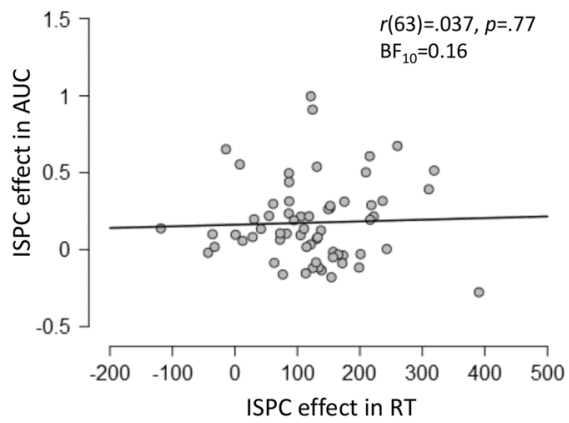

Fig. 5 Scatterplots illustrating the relationship between the item-specific proportion congruency (ISPC) effect as reflected in reaction time (RT) from the key-press version of the Stroop task and the ISPC effects as reflected in initiation time (IT) (panel A), movement time (MT) (panel B), and area under the curve (AUC) (panel C) from the mouse-tracking version of the task 
a Key presses

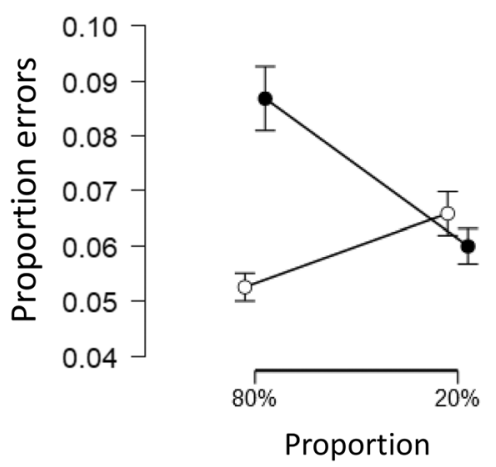

b Mouse movements

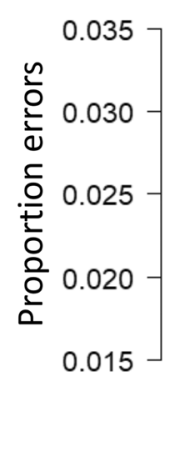

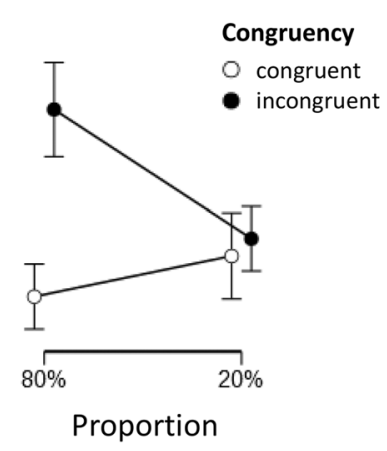

\section{Correlation}

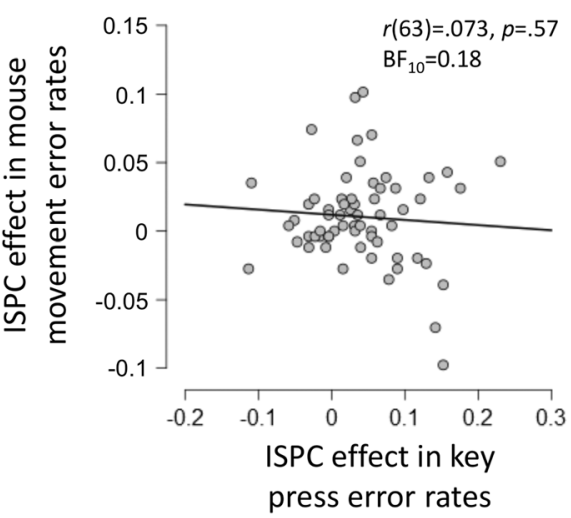

Fig. 6 Error rates for congruent and incongruent trials as a function of proportion in the key-press (panel A) and mouse-tracking version (panel B) of the Stroop task. Error bars represent standard errors. The scatterplot

key-press and mouse-movement responses. In line with previous studies, we found that the ISPC effect could be observed using key presses as well as various mousetracking parameters (cf. Bundt et al., 2018; Grandjean et al., 2013). Moreover, the speed (RT and MT) and movement trajectory (AUC) measurements of the ISPC effect showed a certain internal consistency within each task in that individual differences in the size of the effect were correlated across odd and even blocks. However, we found no evidence that individual differences in the size of the ISPC effect were correlated between tasks, suggesting that conflict adaptation is relatively independent across response modalities.

Our findings contribute to our understanding of individual differences in cognitive control abilities by revealing that the context-sensitivity of conflict control mechanisms (e.g., Abrahamse et al., 2016) also pertains to the task-specific output, in addition to its input. Previous work demonstrated that conflict adaptation is temporally stable (as indicated by strong 2-week test-retest reliability; Clayson \& Larson, 2013; Feldman \& Freitas, 2016), suggesting that an individual's ability to adjust cognitive control settings may be a trait-like characteristic as opposed to a state that fluctuates over time. While intuitively appealing and applicable when repeating the exact same conflict task (input and output are unchanged), it seems that such a top-down view on control mechanisms may be an oversimplification. The current data argue against the idea that some individuals are generally better at adapting their cognitive control settings, and thus against the existence of a domain-general mechanism that is relatively independent of conflict or response type (cf. conflictmonitoring hypothesis; Botvinick et al., 2001). Rather, they support the notion that response modality plays a more integrative role in adaptive control processes, consistent with associative learning models of cognitive in panel $\mathbf{C}$ shows the relationship between the item-specific proportion congruency (ISPC) as reflected in the proportion of errors in the key-press and mouse-tracking versions of the task

control. Our results extend previous indications that adaptation is domain-specific. For example, prior work has shown that conflict control does not generally transfer from one type of conflict to the other within the same task (Funes, Lupiáñez, \& Humphreys, 2010; Notebaert $\&$ Verguts, 2008). In addition, individual differences in conflict control were uncorrelated across different conflict tasks that required the same response modality (e.g., Keye et al., 2009, 2013; Whitehead et al., 2019). Here, we demonstrate that control is also response-specific, as individual differences were uncorrelated across two versions of the same conflict task when different response modalities are required. This further extends insights on the response-specificity of control from previous observations that control does not transfer between response modalities that are intermixed within a conflict task (Braem et al., 2011; Janczyk \& Leuthold, 2018) by demonstrating that adaptive control mechanisms also depend on the response modality when the various modalities are clearly separated.

Our results have implications for work in the cognitive control domain. Many current behavioral and imaging studies are still based on the assumption that conflict control across both different conflict tasks (input) with different response modalities (output) relies on the same or overlapping cognitive control process (e.g., Chen et al., 2018). While it was already demonstrated that these control mechanisms differ as a function of task-input (Keye et al., 2009, 2013; Whitehead et al., 2019), here we show for the first time that processes may even differ for a single conflict task when different response modalities are used. This raises the question of to what extent conclusions from studies involving conflict tasks performed with key presses and mouse movements can be compared, and what mouse-tracking studies add to our knowledge on control as revealed by key-press studies. We believe that 
the merit of mouse-tracking studies is that they provide insight in the dynamics of control, and thus are very useful for gaining a better understanding of cognitive control and its underlying processes. For example, Scherbaum, Dshemuchadse, Fischer, and Goschke (2010) used mouse tracking in a flanker task and revealed that responses on the previous trial influenced the early phase of conflict resolution, whereas the degree of previous and current conflict influenced later phases. In addition, our group has previously used mouse tracking in a Stroop task and demonstrated that adaptive control mechanisms bias actions before and after movement initiation (Bundt et al., 2018). In all, we thus believe that mousetracking and key-press studies can be complementary, as mouse tracking can reveal fundamental insights that otherwise would remain obscured by key-press responses. We speculate that conclusions may be generalized to the other modality at the group level, but researchers should be wary that performance may not necessarily reflect stable, trait-like abilities at the individual level. Challenging the traditional conflict-monitoring account and associative learning models, Schmidt (2013) proposed that the ISPC effect reflects simple learning of predictive relationships (i.e., contingencies) between stimulus features and responses rather than conflict adaptation. Admittedly, the ISPC manipulation in the present study could be argued to be explained by contingency learning alone. As contingency learning requires the development of S-R relationships that facilitate responding, the contingencies may only be implemented in later stages of the task and as such could to some extent be response-specific. Regardless of whether the observed ISPC effects resulted from contingency learning (Schmidt, 2013), conflict-mediated associative learning (Abrahamse et al., 2016; Verguts \& Notebaert, 2009), or a combination of the two, the present results demonstrate the response-specificity of adaptive control mechanisms and argue against the view of adaptation being a domain-general trait.

More broadly, our findings are consistent with seminal studies showing that responses tend to bind to specific stimuli (Bertelson, 1965) or task sets (Rogers \& Monsell, 1995), and demonstrate that response modalities can also bind to certain control or contingency learning strategies. In a similar vein, studies from the motor-learning domain have shown that contextual variations during the learning of new motor chunks or response sequences can have a detrimental effect on motor skill performance, again suggesting a tight link between the action plans and the stimuli on which they are performed (e.g., Ruitenberg, Abrahamse, De Kleine, \& Verwey, 2012; Ruitenberg, Verwey, \& Abrahamse, 2015). In addition, there are indications that motor sequence representations are effector-specific (e.g., De De Kleine \& Verwey, 2009; but see Barnhoorn, Döhring, Van Asseldonk, \& Verwey, 2016), suggesting that motor control may also involve responsespecific mechanisms.

Our study has some limitations that should be acknowledged. First, while our results of both the traditional and Bayesian approaches argue in favor of the null hypothesis, we cannot conclusively refute the possibility that there may have been some relation between conflict control across our tasks. For example, performance could to some extent still rely on shared processes that we were unable to capture here (e.g., general attentional or inhibitory processes). Nonetheless, based on our observation that the non-significant betweentask correlations are very low compared to the significant within-task correlations, we are confident that our conclusion that conflict control as reflected in the ISPC effect is relatively independent across response modalities is warranted. Another limitation is that the mouse movements used in the present study allowed for more online corrections in case an incorrect response was "selected," while this is much more difficult with key-press responses. One could wonder if stronger associations could have been obtained when using other response modalities that also allow for corrections (e.g., verbal response).

Overall, the present study demonstrates that individual differences in the ISPC effect are uncorrelated across key presses and mouse movements, indicating that different control mechanisms drive adaptation performance depending on the response modality. Our findings in combination with the literature suggest that control may be rooted in both task-specific perceptual input and motor output, as also argued by associative models of cognitive control (Abrahamse et al., 2016). Future studies should systematically manipulate both response modality (output; present study), stimulus modality (e.g., auditory vs. visual; cf. Kreutzfeldt, Stephan, Willmes, \& Koch, 2016), and conflict type (input; cf. Keye et al., 2009, 2013; Whitehead et al., 2019) in a single design to further elucidate the respective contribution of each factor to the specificity of conflict control.

Acknowledgements MR was supported by a postdoctoral fellowship from the Ghent University Special Research Fund (BOF 15/PDO/135).

Open Practice Statement The data that support the findings of this study are available on request from the corresponding author [M.R.]. The experiment was not preregistered.

\section{References}

Abrahamse, E., Braem, S., Notebaert, W., \& Verguts, T. (2016). Grounding cognitive control in associative learning. Psychological Bulletin, 142, 693-728.

Abrahamse, E. L., Duthoo, W., Notebaert, W., \& Risko, E. F. (2013). Attention modulation by proportion congruency: The asymmetrical list shifting effect. Journal of Experimental Psychology: Learning, Memory, and Cognition, 39, 1552-1562. 
Annett, M. (1970). A classification of hand preference by association analysis. British Journal of Psychology, 61, 303-321.

Barnhoorn, J. S., Döhring, F. R., Van Asseldonk, E. H., \& Verwey, W. B. (2016). Similar representations of sequence knowledge in young and older adults: A study of effector independent transfer. Frontiers in Psychology, 7: 1125.

Bertelson, P. (1965). Serial choice reaction-time as a function of response versus signal-and-response repetition. Nature, 205, 217-218.

Botvinick, M. M., Braver, T. S., Barch, D. M., Carter, C. S., \& Cohen, J. D. (2001). Conflict monitoring and cognitive control. Psychological Review, 108, 624-652.

Botvinick, M. M., Cohen, J. D., \& Carter, C. S. (2004). Conflict monitoring and anterior cingulate cortex: An update. Trends in Cognitive Sciences, 8, 539-546.

Braem, S., Abrahamse, E. L., Duthoo, W., \& Notebaert, W. (2014). What determines the specificity of conflict adaptation? A review, critical analysis, and proposed synthesis. Frontiers in Psychology, 5: 1134.

Braem, S., Verguts, T., \& Notebaert, W. (2011). Conflict adaptation by means of associative learning. Journal of Experimental Psychology: Human Perception and Performance, 37, 1662-1666.

Bugg, J. M., \& Crump, M. J. C. (2012). In support of a distinction between voluntary and stimulus-driven control: A review of the literature on proportion congruent effects. Frontiers in Psychology, 3: 367.

Bundt, C., Ruitenberg, M. F. L., Abrahamse, E. L., \& Notebaert, W. (2018). Early and late indications of item-specific control in a Stroop mouse tracking study. PLoS ONE, 13: e0197278.

Chen, T., Becker, B., Camilleri, J., Wang, L., Yu, S., Eickhoff, S. B., \& Feng, C. (2018). A domain-general brain network underlying emotional and cognitive interference processing: Evidence from coordinate-based and functional connectivity meta-analyses. Brain Structure and Function, 223, 3813-3840.

Clayson, P. E. \& Larson, M. J. (2013). Psychometric properties of conflict monitoring and conflict adaptation indices: Response time and conflict N2 event-related potentials. Psychophysiology, 50, 1209-1219.

Cohen, J. (1988). Statistical power analysis for the behavioral sciences (2nd ed.). Hillsdale: Lawrence Erlbaum Associates.

IBM Corp (2015). IBM SPSS Statistics for Windows (Version 23.0) [Computer software].

De Kleine, E., \& Verwey, W. B. (2009). Representations underlying skill in the discrete sequence production task: Effect of hand used and hand position. Psychological Research, 73, 685-694.

Dienes, Z. (2011). Bayesian versus orthodox statistics: Which side are you on? Perspectives on Psychological Science, 6, 274-290.

Donohue, S. E., Liotti, M., Perez, R., \& Woldorff, M. G. (2012). Is conflict monitoring supramodal? Spatiotemporal dynamics of cognitive control processes in an auditory Stroop task. Cognitive, Affective, \& Behavioral Neuroscience, 12, 1-15.

Duthoo, W., Abrahamse, E. L., Braem, S., Boehler, C. N., \& Notebaert, W. (2014). The heterogeneous world of congruency sequence effects: an update. Frontiers in Psychology, 5: 1001.

Egner, T. (2014). Creatures of habit (and control): A multi-level learning perspective on the modulation of congruency effects. Frontiers in Psychology, 5: 1247.

Faulkenberry, T. J., Cruise, A., Lavro, D., \& Shaki, S. (2016). Response trajectories capture the continuous dynamics of the size congruity effect. Acta Psychologica, 163, 114-123.

Feldman, J. L., \& Freitas, A. L. (2016). An investigation of the reliability and self-regulatory correlates of conflict adaptation. Experimental Psychology, 63, 237-247.

Freeman, J. B., \& Ambady, N. (2009). Motions of the hand expose the partial and parallel activation of stereotypes. Psychological Science, 20, 1183-1188.

Freeman, J. B., \& Ambady, N. (2010). MouseTracker: Software for studying real-time mental processing using a computer mousetracking method. Behavior Research Methods, 42, 226-241.
Funes, M. J., Lupiáñez, J., \& Humphreys, G. (2010). Sustained vs. transient cognitive control: Evidence of a behavioral dissociation. Cognition, 114, 338-347.

Geukes, S., Gaskell, M. G., \& Zwitserlood, P. (2015). Stroop effects from newly learned color words: Effects of memory consolidation and episodic context. Frontiers in Psychology, 6: 278.

Grandjean, J., D’Ostilio, K., Fias, W., Phillips, C., Balteau, E., Degueldre, C., ... Collette, F. (2013). Exploration of the mechanisms underlying the ISPC effect: Evidence from behavioral and neuroimaging data. Neuropsychologia, 51, 1040-1049.

Incera, S., Markis, T. A., \& McLennan, C. T. (2013). Mouse-tracking reveals when the Stroop effect happens. The Ohio Psychologist, 60, 33-34.

Jacoby, L. L., Lindsay, D. S., \& Hessels, S. (2003). Item-specific control of automatic processes: Stroop process dissociations. Psychonomic Bulletin \& Review, 10, 638-644.

Janczyk, M., \& Leuthold, H. (2018). Effector system-specific sequential modulations of congruency effects. Psychonomic Bulletin \& Review, $25,1066-1072$

JASP Team (2017). JASP (Version 0.8.1) [Computer software].

Keye, D., Wilhelm, O., Oberauer, K., \& Stürmer, B. (2013). Individual differences in response conflict adaptations. Frontiers in Psychology, 4: 947.

Keye, D., Wilhelm, O., Oberauer, K., \& van Ravenzwaaij, D. (2009). Individual differences in conflict-monitoring: Testing means and covariance hypothesis about the Simon and the Eriksen Flanker task. Psychological Research, 73, 762-776.

Kreutzfeldt, M., Stephan, D. N., Willmes, K., \& Koch, I. (2016). Shifts in target modality cause attentional reset: Evidence from sequential modulation of crossmodal congruency effects. Psychonomic Bulletin \& Review, 23, 1466-1473.

Laird, A. R., McMillan, K. M., Lancaster, J. L., Kochunov, P., Turkeltaub, P. E., Pardo, J. V., \& Fox, P. T. (2005). A comparison of label-based review and ALE meta-analysis in the Stroop task. Human Brain Mapping, 25, 6-21.

MacLeod, C. M. (1991). Half a century of research on the Stroop effect: An integrative review. Psychological Bulletin, 109, 163-203.

Notebaert, W., \& Verguts, T. (2008). Cognitive control acts locally. Cognition, 106, 1071-1080.

Nuzzo, R. L. (2017). An introduction to Bayesian data analysis for correlations. $P M \& R$, 9, 1278-1282.

Rogers, R. D., \& Monsell, S. (1995). Costs of a predictable switch between simple cognitive tasks. Journal of Experimental Psychology: General, 124, 207-231.

Ruitenberg, M. F. L., Abrahamse, E. L., De Kleine, E., \& Verwey, W. B. (2012). Context-dependent motor skill: Perceptual processing in memory-based sequence production. Experimental Brain Research, 222, 31-40.

Ruitenberg, M. F. L., Abrahamse, E. L., Santens, P., \& Notebaert, W. (2019). The effect of dopaminergic medication on conflict adaptation in Parkinson's disease. Journal of Neuropsychology, 13, 121135.

Ruitenberg, M. F. L., Verwey, W. B., \& Abrahamse, E. L. (2015). What determines the impact of context on sequential action? Human Movement Science, 40, 298-314.

Scherbaum, S., Dshemuchadse, M., Fischer, R., \& Goschke, T. (2010). How decisions evolve: The temporal dynamics of action selection. Cognition, 115, 407-416.

Schmidt, J. R. (2013). The parallel episodic processing (PEP) model: Dissociating contingency and conflict adaptation in the itemspecific proportion congruent paradigm. Acta Psychologica, 142, 119-126.

Verguts, T., \& Notebaert, W. (2008). Hebbian learning of cognitive control: Dealing with specific and nonspecific adaptation. Psychological Review, 115, 518-525. 
Verguts, T., \& Notebaert, W. (2009). Adaptation by binding: A learning account of cognitive control. Trends in Cognitive Sciences, 13, 252257

Weekes, N. Y., \& Zaidel, E. (1996). The effects of procedural variations on lateralized Stroop effects. Brain and Cognition, 31, 308-330.

Weissman, D. H., Colter, K., Drake, B., \& Morgan, C. (2015). The congruency sequence effect transfers across different response modes. Acta Psychologica, 161, 86-94.

Whitehead, P. S., Brewer, G. A., \& Blais, C. (2019). Are cognitive control processes reliable? Journal of Experimental Psychology: Learning, Memory, and Cognition, 45, 765-778.
Yamamoto, N., Incera, S., \& McLennan, C. T. (2016). A reverse Stroop task with mouse tracking. Frontiers in Psychology, 7: 670.

Publisher's note Springer Nature remains neutral with regard to jurisdictional claims in published maps and institutional affiliations. 